\title{
Robotic right upper lobectomy: Twelve steps
}

\author{
Prabhu Sasankan, BS, ${ }^{\mathrm{a}}$ Stephanie Chang, MD, ${ }^{\mathrm{b}}$ and Robert Cerfolio, MD, MBA, ${ }^{\mathrm{b}}$ New York, NY
}

\footnotetext{
From the ${ }^{\mathrm{a}}$ New York University Grossman School of Medicine and ${ }^{\mathrm{b}}$ Division of Thoracic Surgery, Department of Cardiothoracic Surgery, NYU Langone Health, New York, NY.

Disclosures: Dr Cerfolio has no disclosures over the past two years but had previous disclosed relationships with AstraZeneca, Bard Davol, Bovie Medical Corporation, C-SATS, ConMed, Covidien/Medtronic, Ethicon, Fruit Street Health, Google/Verb Surgical, Intuitive Surgical, KCI/Acelity, Myriad Genetics, Neomend, Pinnacle Biologics, ROLO-7, Tego, and TransEnterix. Mr Sasankan and Dr Chang reported no conflicts of interest.

The Journal policy requires editors and reviewers to disclose conflicts of interest and to decline handling or reviewing manuscripts for which they may have a conflict of interest. The editors and reviewers of this article have no conflicts of interest.

Received for publication Oct 20, 2020; revisions received Jan 20, 2021; accepted for publication Jan 27, 2021; available ahead of print Feb 12, 2021

Address for reprints: Robert Cerfolio, MD, MBA, New York University Langone Health, 550 1st Ave, 15th Floor, New York, NY 10016 (E-mail: robert.cerfolio@nyulangone.org).

JTCVS Techniques 2021;7:280-2

2666-2507

Copyright (C) 2021 The Authors. Published by Elsevier Inc. on behalf of The American Association for Thoracic Surgery. This is an open access article under the CC BY-NC-ND license (http://creativecommons.org/licenses/bync-nd/4.0/).

https://doi.org/10.1016/j.xjtc.2021.01.043
}

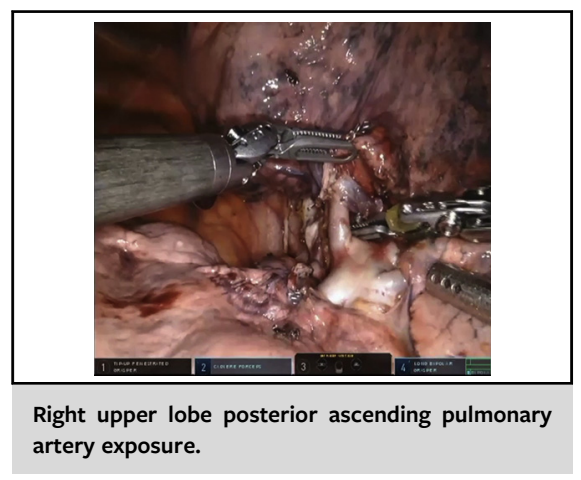

CENTRAL MESSAGE

A posterior-to-anterior approach for a robotic right upper lobectomy is a safe and efficient method for resection.

See Commentary on page 283
Video clip is available online.

Feature Editor's Introduction-Welcome to the beginning of a series of new format articles in JTCVS Techniques termed Video Atlas Articles (VAAs). VAAs are peerreviewed descriptions of a surgical procedure crystallized into a series of steps, with each step taught through its own narrated video and corresponding short text. The purpose of the VAA series is to provide continuing surgical education in an enduring format. Quality is maintained by the invitation of expert authors and by the iterative peer-review process involving expert reviewers. Thoracic surgeons are privileged to care for patients with a vast breadth and depth of conditions using an expansive armamentarium of rapidly evolving techniques. Most thoracic surgeons will perform operations they had never before performed in their training, and many will become expert in them. In fact, an entire generation of thoracic surgeons learned robotic thoracic surgery without residency or fellowship training on the robot, and surgeons from this generation are the current leaders in robotic thoracic surgery. This field is fastpaced and rapidly evolving. To provide the highest level of care to our patients, to embrace (and drive) the exciting innovation in our field, and sometimes even to remain relevant in the field of thoracic surgery requires the surgeon's effort in self-learning. There are many modalities for continuing surgical education; however, with regard to technique, there is little substitute for the first-hand experience of observing an expert surgeon operating, for junior and seasoned surgeons alike. The VAA is designed to replicate this experience in a quality-assured, on-hand, and high-impact format. Robotic right upper lobectomy is an index operation of the modern thoracic surgeon, and herein it is professionally illustrated in a VAA.

\section{Bryan M. Burt, MD}

The right upper lobectomy is a standard minimally invasive surgical procedure. Here we describe our process for performing the right upper lobectomy using a posteriorto-anterior approach. Patient consent was attained for all the patient data and media used in this report.

\section{METHODS}

Our technique for robotic right upper lobectomy is described in 12 steps, each with an accompanying video. This described posterior-to-anterior approach uses the da Vinci Xi system (Intuitive Surgical, Sunnyvale, Calif).

The robotic arm configuration, with associated instrumentation, is as follows:

Robotic arm 1: tip-up fenestrated grasper

Robotic arm 2: cadiere forceps

Robotic arm 3: 0-degree robotic camera

Robotic arm 4: long bipolar grasper (with energy) or robotic stapler 


\section{RESULTS}

Single lung isolation is achieved with a double-lumen endotracheal tube. The patient is placed in the left lateral decubitus position. Care is taken to adequately pad the patient's arms and legs, to buffer any zone against which the patient's body will be pressed. ${ }^{1}$ A mild degree of flexion is then introduced to the operating table to increase the space available between the ribs and to displace the hip from the chest. No arterial line or urinary catheter is placed for the procedure.

The right upper lobectomy is performed in the following 12 steps:

1. Port placement (Video 1). The scapula is identified, and the edge is marked. Next, the midpoint of the spine is palpated, and a line is then drawn along the vertebral right lateral pedicles. The ribs are counted, and the top of the ninth rib is marked from anterior to posterior. Incision sites for robotic arms 1, 2, and 3 are then marked along the line of the ninth rib, measured from its intersection with the right lateral pedicle. The site for robotic arm 1 (8-mm port) is marked $4 \mathrm{~cm}$ from the right lateral pedicle. Robotic arm $2(8 \mathrm{~mm})$ is placed $8 \mathrm{~cm}$ medial to robotic arm 1 , and the camera port is placed $8 \mathrm{~cm}$ medial to robotic arm 2. Occasionally, a smaller patient size may necessitate adjusting the spacing of these ports (such as $2 \mathrm{~cm}, 7 \mathrm{~cm}$, and $7 \mathrm{~cm}$ ). An incision is made at the camera port site marking, and a trocar is placed, followed by insertion of the thoracoscopic camera. Once visualization of the thoracic cavity is ensured, insufflation is begun. Under thoracoscopic visualization, preemptive analgesia is administered via subpleural injection of local anesthetic at the remaining planned incision sites, and a concurrent intercostal paraspinal block is performed. Subsequent ports are placed under visualization by the camera. A 12$\mathrm{mm}$ port for robotic arm 4 is placed anteriorly in the seventh interspace, avoiding the rectus muscles and placed just above the diaphragm. Finally, the assistant port is placed inferior and between the camera port and the port for robotic arm 4 , creating a triangle configuration.

2. Dissection of inferior pulmonary ligament and removal of station 8 and 9 lymph nodes (Video 2). Robotic arm 1 is used to retract the lung to expose the inferior pulmonary ligament. On entry, the chest is explored to ensure the absence of metastatic lesions. The inferior pulmonary ligament is taken down, and the station 8 and 9 lymph nodes are removed.

3. Dissection of station 7 lymph nodes (Video 3). The station 7 subcarinal lymph node packet is dissected. Care is taken to ensure hemostasis as this entire lymph node packet is removed. Importantly, we use robotic

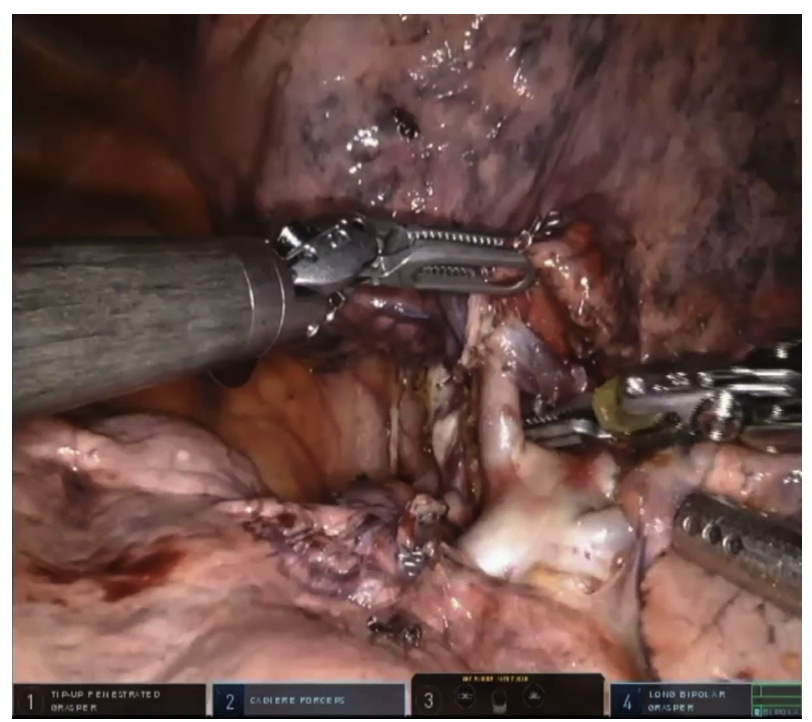

FIGURE 1. The posterior ascending artery (A2) is identified prior to transection.

arm 1 to simply push against the superior segment (S6) to retract it anteriorly and expose the lung. We avoid closing our instrument against the lung here, as doing so can lead to tearing and air leaks. Robotic arm 1 may also assist in retracting the posterior segment (S2) in this manner if needed.

4. Dissection of station 11 lymph node (Video 4). Once the entire subcarinal lymph node packet is removed, locating the posterior vein (V2) is critical. The bronchus can be dissected to identify and distinguish the right upper lobe takeoff from the right mainstem bronchus. The station 11 lymph node is located under the right upper lobe and at the proximal part of the bronchus intermedius. The station 11 node must be taken carefully because the pulmonary artery lies just below this node, as does the V2 vein.

5. Completion of the posterior fissure (Video 5). In a patient with a complete or near-complete fissure, the fissure can be carefully dissected to identify the anatomy within. The V2 vein and posterior ascending pulmonary artery (A2) and superior segment pulmonary artery (A6) branches, as well as any aberrant veins crossing the fissure, should be identified. The posterior fissure can then be taken carefully.

6. Dissection and division of the posterior ascending pulmonary artery (Video 6). The A2 artery neck is identified, as is the V2 vein coursing above it (Figure 1). The A2 is taken with a vascular stapler.

7. Dissection and division of the right upper lobe bronchus (Video 7). The right upper lobe bronchus is dissected off the underlying 10R lymph node and the truncus artery (A1/A3). The bronchus is retracted, and the dissection is carried out just under the 
bronchus, removing all number 10 lymph nodes. The bronchus is freed from the underlying anatomy. The entire 10R lymph node packet can subsequently be resected at this time. It is important at this step to carefully dissect the pulmonary artery off the anterior surface of the bronchus to provide a clear window for dissection.

8. Dissection and division of the truncus arteriosus (Video 8). Once the anterior (A3) and apical (A1) pulmonary artery, also known as the truncus arteriosus, are identified, the surgeon can choose to take them separately or together. The azygos vein should be carefully identified during this part of the procedure. We prefer to hold the divided bronchus with robotic arm 2, exposing the $\mathrm{V} 2$ vein, azygos vein, and $\mathrm{A} 1$ and $\mathrm{A} 3$ branches. The arterial branches can then be stapled with a vascular stapler.

9. Dissection and division of the upper lobe vein (Video 9). We prefer to take the upper lobe vein via a posterior approach. To accomplish this, continue to retract the lung anteriorly; robotic arm 4 can be used to hold the lung in this retracted position. By doing so, access to the upper lobe vein can be achieved; there is no need to retract the lung posteriorly to complete this step with an anterior approach. We take the vein off the 10R lymph node, with care taken to identify and avoid the phrenic nerve. The superior vena cava and azygos junction are identified, and the number 10 lymph nodes are completely removed. The entire V2 vein can then be taken. V1 and V3 are often completely encompassed within V2; if not, they can be taken during completion of the anterior fissure.

10. Dissection of station 2 and 4 lymph nodes (Video 10 ). It is critical that in every case of confirmed or suspected malignancy, a complete thoracic lymphadenectomy is performed. The innominate artery and superiormost recess of the pericardium are identified. The trachea is then identified, and care is taken to preserve the vagus nerve as the $2 \mathrm{R}$ and $4 \mathrm{R}$ lymph node packets are dissected from the trachea. Additional remaining number 10 lymph nodes can be taken at this time by lifting the azygos to reveal these nodes abutting the pulmonary artery. We perform this standard lymph node dissection in all patients, irrespective of the size or positron emission tomography activity of their nodule.

11. Completion of the anterior fissure (Video 11). Although it is common to attempt this step from an anterior approach, fully elevating the anteriormost aspect of the right upper lobe can be difficult when attempting to completely remove through with such an approach. For this reason, we prefer to take the fissure posteriorly. A posterior approach also allows for taking the fissure while continuing to retract the lung anteriorly, thereby allowing for less repositioning of the lung. During completion of the fissure, it is important to ensure that the transected hilar structures of the right upper lobe are above the staple line.

12. Specimen removal (Video 12). The bag is placed through the anterior port. While the bag is being deployed in the chest, robotic arm 1 is used to pull the corner of the bag inferiorly to help widely open the bag. Robotic arms 1 and 2 are used to place the specimen in the bag. Before pulling out the bag, we ensure that it is not caught on any staple lines. Hemostasis is obtained, with careful examination of all port sites. A $20 \mathrm{Fr}$ chest tube is placed into the chest, and the lung is reexpanded under direct visualization.

\section{DISCUSSION}

Minimally invasive approaches to lobectomy have been associated with improved clinical effectiveness, reduced perioperative morbidity, and similar total perioperative cost relative to an open thoracotomy approach. ${ }^{3}$ Robotic thoracic surgery in particular offers the advantage of improved visualization, ergonomics, and precision of movement. The robotic approach to lobectomy has been shown to result in excellent lymph node dissection, minimal length of stay, and minimal pain. ${ }^{4}$ Comparison of quality outcome measures between robotic and VATS approaches have demonstrated robotic surgery to be equivalent or even potentially superior to VATS.,

In conclusion, these 12 steps outline a posterior-toanterior approach to a robotic right upper lobectomy with mediastinal lymph node dissection. In our opinion, this order of steps yields an efficient and high-quality operation.

\section{References}

1. Cerfolio RJ, Steenwyk BL, Watson C, Sparrow J, Belopolsky V, Townsley M, et al. Decreasing the preincision time for pulmonary lobectomy: the process of lean and value stream mapping. Ann Thorac Surg. 2016;101:1110-5.

2. Geraci TC, Sasankan P, Luria B, Cerfolio RJ. Intraoperative anesthetic and surgical concerns for robotic thoracic surgery. Thorac Surg Clin. 2020;30:293-304.

3. Kneuertz PJ, Singer E, D'Souza DM, Abdel-Rasoul M, Moffatt-Bruce SD, Merritt RE. Hospital cost and clinical effectiveness of robotic-assisted versus video-assisted thoracoscopic and open lobectomy: a propensity score-weighted comparison. J Thorac Cardiovasc Surg. 2019;157:2018-26.

4. Nasir BS, Bryant AS, Minnich DJ, Wei B, Cerfolio RJ. Performing robotic lobectomy and segmentectomy: cost, profitability, and outcomes. Ann Thorac Surg. 2014;98:203-8; discussion 208-9.

5. Louie BE, Wilson JL, Kim S, Cerfolio RJ, Park BJ, Farivar AS, et al. Comparison of video-assisted thoracoscopic surgery and robotic approaches for clinical stage I and stage II non-small cell lung cancer using the Society of Thoracic Surgeons database. Ann Thorac Surg. 2016;102:917-24.

6. Farivar AS, Cerfolio RJ, Vallières E, Knight AW, Bryant A, Lingata V, et al. Comparing robotic lung resection with thoracotomy and video-assisted thoracoscopic surgery cases entered into the Society of Thoracic Surgeons database. Innovations. 2014;9:10-5. 